\section{REAL-WORLD PATTERNS OF CARE AND OUTCOMES IN PATIENTS WITH MICROSATELLITE-INSTABILITY HIGH TUMORS IN ADVANCED ENDOMETRIAL CANCER IN THE UNITED STATES}

'S Kelkar, ${ }^{1} \mathrm{~S}$ Corman, ${ }^{2} \mathrm{C}$ Macahilig, ${ }^{3} \mathrm{~V}$ Prabhu*, ${ }^{1} \mathrm{~N}$ Rusibamayila, ${ }^{2} \mathrm{~S}$ Odak, ${ }^{3} \mathrm{R}$ Orlowski, ${ }^{4} \mathrm{~L}$ Duska*. ${ }^{1}$ OPEN Health, Evidence and Access, Bethesda, MD, USA; ${ }^{2}$ RTI-Health Solutions, Surveys and Observational Studies, Research Triangle, NC, USA; ${ }^{3}$ Merck and Co., Inc., Outcomes Research (Oncology-Cervical and Endometrial), Kenilworth, NJ, USA; ${ }^{4}$ University of Virginia Health System, Gynecologic Oncology Division Department of Obstetrics and Gynecology, Charlottesville, VA, USA

\subsection{6/ijgc-2021-ESGO.186}

Introduction/Background* New/emerging therapies based on microsatellite instability (MSI) prognostic biomarker are altering the treatment landscape for advanced endometrial cancer (aEC). The real-world outcomes in aEC patients with MSIhigh/deficient mismatch repair (MSI-H/dMMR) tumor status is not well understood.

Methodology Endometrial Cancer Health Outcomes (ECHO) is a multicenter retrospective chart review study in women diagnosed with aEC in the US. Physicians extracted de-identified data from medical records of women ( $\geq 18$ years-old) diagnosed with inoperable aEC, with known MSI/MMR status, who progressed after prior systemic therapy between 07/01/ 2016-07/30/2019. Data included patient demographics, clinical/ treatment characteristics, and clinical outcomes. Kaplan-Meier analyses were performed to estimate time to treatment discontinuation, real-world progression-free survival (rwPFS) and overall survival (OS). The study was IRB approved.

Result(s)* Among 130 MSI-H/dMMR aEC patients who progressed on a prior systemic therapy, mean age was 62 years, $57 \%$ had endometrioid carcinoma histology, and 93\% had ECOG 0-1. As $2^{\text {nd }}$-line therapy, $79 \%$ of patients received immunotherapy (IO) \pm chemotherapy (CT) (pembrolizumab monotherapy $=71 \%), 14 \%$ received CT only, and 7\% received hormonal or other therapy (HT/OT). Median duration of $2^{\text {nd }}$ line therapy was 14 months. Median rwPFS from initiation of $2^{\text {nd }}$-line therapy was 17 months $(95 \%$ confidence interval [CI]: 13-n/a) [IO \pm CT: 29 months (95\% CI: 18-n/a); CT: 4 months (95\% CI: 2-9)]. Median OS from $2^{\text {nd }}$-line therapy initiation was 30 months (95\% CI: 18-n/a) $[\mathrm{IO} \pm \mathrm{CT}$ : median not reached (95\% CI: 30-n/a); CT: 5.5 months (95\% CI: 4-n/a)]. (Table 1)
Conclusion* In real-world practice, most MSI-high/dMMR aEC patients in the US who failed a prior systemic therapy, initiated immunotherapy with favorable outcomes.

\section{BIOMARKER ANALYSIS OF THE PHASE 2 STUDY OF PEMBROLIZUMAB IN COMBINATION WITH DOXORUBICIN IN ADVANCED ENDOMETRIAL CANCER: TOPIC TRIAL/VHIO10001}

${ }^{1} \mathrm{JM}$ Piulats* ${ }^{*}{ }^{2} \mathrm{~L}$ Fariñas-Madrid, ${ }^{3} \mathrm{M}$ Santacana, ${ }^{4} \mathrm{MJ}$ Rubio Pérez, ${ }^{5} \mathrm{~A}$ Redondo ${ }^{6} \mathrm{G}$ Villacampa, ${ }^{7} \mathrm{~A}$ Yubero, ${ }^{8} \mathrm{I}$ Romero, ${ }^{1} \mathrm{M}$ Gil-Martin, ${ }^{9} \mathrm{~J}$ Garcia-Donas, ${ }^{10} \mathrm{~A}$ GonzalezMartin, ${ }^{3} \mathrm{~S}$ Gatius, ${ }^{5} \mathrm{~A}$ Gallego Martínez, ${ }^{2} \mathrm{~F}$ Grau, ${ }^{6} \mathrm{~F}$ Ruiz, ${ }^{1} \mathrm{~B}$ Pardo Búrdalo, ${ }^{11} \mathrm{~L}$ Sánchez Lorenzo, ${ }^{3} \mathrm{X}$ Matias Guiu, ${ }^{2} \mathrm{~A}$ Oaknin. ${ }^{1}$ Institut Català d'Oncologia- L'Hospitalet, Medical Oncology, Barcelona, Spain; ${ }^{2}$ Gynaecologic Cancer Programme Vall d'Hebron Institute of Oncology (VHIO), Hospital Universitari Vall d'Hebron, Vall d'Hebron Barcelona Hospital Campus, Medical Oncology, Barcelona, Spain; ${ }^{3}$ Hospital Universitari Arnau de Vilanova, University of Lleida, IRBLleida, CIBERONC, Department of Pathology and Molecular Genetics and Research Laboratory, Lleida, Spain; ${ }^{4}$ Hospital Universitario Reina Sofía, Medical Oncology, Córdoba, Spain; ${ }^{5}$ Hospital Universitario La Paz, Medical Oncology, Madrid, Spain; ${ }^{6}$ Vall d'Hebron Institut of Oncology (VHIO), Oncology Data Science, Barcelona, Spain; ${ }^{7}$ Hospital Clínico Universitario Lozano Blesa, Medical Oncology, Zaragoza, Spain; ${ }^{8}$ Fundación Instituto Valenciano de Oncología, Medical Oncology, Valencia, Spain; ${ }^{9}$ Hospital Madrid Norte San Chinarro Centro Integral Oncologico Clara Campal, Genitourinary, Ginecologycal, skin and rare tumors Unit, Madrid, Spain; ${ }^{10} \mathrm{Grupo}$ Español de Investigación en Cáncer de Ovario (GEICO), Clínica Universidad de Navarra, Medical Oncology, Madrid Spain; ${ }^{11}$ Clínica Universidad de Navarra, Medical Oncology, Madrid, Spain

\subsection{6/ijgc-2021-ESGO.187}

Introduction/Background* Pembrolizumab conferred a 57\% objective response rate (ORR) in patients with mismatch repair deficient (MMRd) advanced endometrial cancer (EC). However, the majority of patients with advanced disease have no specific molecular profile (NSMP) tumours or p53-abnormal (p53abn). We hypothesized that pembrolizumab with an inducer of immunogenic cell death such as doxorubicin could improve clinical responses and survival in all EC molecular subtypes.

Methodology The TOPIC study (NCT03276013) was an investigator-initiated, single-arm, multi-centre, phase II study in patients with recurrent/metastatic EC who received one prior line of platinum-based chemotherapy. Patients received doxorubicin $60 \mathrm{mg} / \mathrm{m}^{2}$ IV every 3 weeks for up to 9 cycles in combination with pembrolizumab $200 \mathrm{mg}$ IV every 3 weeks up to 36 cycles, or until progression or toxicity. Tumour samples

Abstract 722 Table 1 Time to Treatment discontinuation, overall survival, and real-world progression free survival in MSI-High or dMMR aEC patients since initiation of $2^{\text {nd }}$ line of therapy overall and stratified by treatment category

\begin{tabular}{|c|c|c|c|c|c|}
\hline & $\begin{array}{l}\text { Overall } \\
(\mathrm{N}=130)\end{array}$ & $\begin{array}{l}\text { Immunotherapy } \pm \\
\text { chemotherapy } \\
(\mathrm{N}=103)\end{array}$ & $\begin{array}{l}\text { Hormonal Therapy } \\
(\mathrm{N}=5)\end{array}$ & $\begin{array}{l}\text { Chemotherapy } \\
(\mathrm{N}=18)\end{array}$ & $\begin{array}{l}\text { Other therapy } \\
(\mathrm{N}=4)\end{array}$ \\
\hline $\begin{array}{l}\text { Time to treatment discontinuation, } \\
\text { median }(95 \% \mathrm{Cl}) \text {, (months) }\end{array}$ & $14.0(9.0,23.0)$ & $21.0(15.0,)$. & $6.0(5.0,15.0)$ & $4.0(2.0,6.0)$ & $7.0(3.0,9.0)$ \\
\hline Probability at 6 months (\%) & $67.7(60.1,76.2)$ & $78.6(71.1,87)$ & $40(13.7,100)$ & $16.7(5.9,46.8)$ & $50.0(18.8,100)$ \\
\hline Probability at 12 months (\%) & $57.7(49.8,66.8)$ & $62.9(54.2,73)$ & $20(3.5,100)$ & $11.1(3.0,41)$ & $0(0,0)$ \\
\hline Probability at 24 months (\%) & $37.3(29,48)$ & $47.4(37.7,59.5)$ & $0(0,0)$ & $0(0,0)$ & $0(0,0)$ \\
\hline $\begin{array}{l}\text { Overall survival, median (95\% Cl), } \\
\text { (months) }\end{array}$ & $30.0(18.0,)$. &.$(30.0,)$. & $18.0(7.0,)$. & $5.5(4.0,)$. & $12.5(10.0,)$. \\
\hline Probability at 6 months (\%) & $86.1(80.3,92.3)$ & $93.2(88.4,98.2)$ & $80.0(51.6,100)$ & $44.4(26.5,74.5)$ & $100.0(100,100)$ \\
\hline Probability at 12 months (\%) & $79.1(72.4,86.4)$ & $78(70.2,86.6)$ & $60.0(29.3,100)$ & $33.3(17.3,64.1)$ & $50.0(18.8,100)$ \\
\hline Probability at 24 months (\%) & $70.1(62.6,78.6)$ & $61.8(51.5,74.2)$ & $60.0(29.3,100)$ & $33.3(17.3,64.1)$ &.$(.)$. \\
\hline $\begin{array}{l}\text { Real-world progression-free survival, } \\
\text { median }(95 \% \mathrm{Cl}) \text {, (months) }\end{array}$ & $17.0(13.0,)$. & $29.0(18.0,)$. & $6.0(5.0,15.0)$ & $4.0(2.0,9.0)$ & $8.5(3.0,20.0)$ \\
\hline Probability at 6 months (\%) & $73.5(66.3,81.6)$ & $82.2(75.1,90)$ & $40.0(13.7,100)$ & $33.3(17.3,64.1)$ & $75(42.6,100)$ \\
\hline Probability at 12 months (\%) & $63.4(55.5,72.3)$ & $70.2(61.9,79.8)$ & $20.0(3.5,100)$ & $11.1(3.0,41)$ & $25(4.6,100)$ \\
\hline Probability at 24 months (\%) & $58.6(50.6,67.8)$ & $53.5(43.5,65.9)$ & $20.0(3.5,100)$ & $11.1(3.0,41)$ & $0(0,0)$ \\
\hline
\end{tabular}




\begin{tabular}{lllll} 
Abstract 731 Table 1 & & \\
\hline & Total & MMRd $(n=5)$ & NSMP (n=10) & p53abn (n=23) \\
\hline PFS rate at 6m (95\%Cl) & $59 \%(45-78)$ & $53 \%(21-100)$ & $78 \%(55-100)$ & $52 \%(35-77)$ \\
Median PFS & 6.5 months & 6.2 months & 10.3 months & 6.4 months \\
ORR & $32 \%$ & $40 \%$ & $40 \%$ & $26 \%$ \\
OS rate at 18m & $51 \%$ & $25 \%$ & $74 \%$ & $49 \%$ \\
Median OS & 18.5 months & 11.9 months & Not reached & 14.8 months \\
\hline
\end{tabular}

were analysed before treatment. Immunohistochemistry was performed for mismatch repair proteins and $\mathrm{p} 53$. Single-gene sequencing was used to detect polymerase- $\mathrm{C}$ (POLE) exonuclease domain mutations. Primary outcome was progressionfree survival rate at 6 months (PFS6). Subgroups were assessed relative to the primary outcome, ORR, median overall survival (OS), and 18-months OS rate.

Result(s)* Molecular classification could be determined in 44 out of 48 patients included. As defined in the protocol, carcinosarcoma patients $(n=6)$ were considered an exploratory cohort and were excluded from this analysis. In the non-carcinosarcoma cohort $(\mathrm{n}=38)$ median age was 66 years (range 37-80). Five (11.4\%) patients were MMRd, 10 (22.7\%) NSMP and $23(52.3 \%)$ p53abn. No patient with POLEmutated tumour was enrolled. Fifty-eight patients had endometroid EC, $42 \%$ serous carcinoma. Histologic grade at diagnosis: grade $1,12.2 \%$; grade $2,21.1 \%$ and grade $3,44.6 \%$. Median follow-up at data cut-off was 19.1 months. Overall PFS6 was 59.3\% (95\%CI 45.4\%-77.6\%) with a median PFS of 6.5 months. Efficacy data according to patients' molecular subtype are shown (see table 1). Overall, the NSMP subtype showed the better efficacy results (PFS6 of $78 \%$ and $40 \%$ ORR).

Conclusion* Pembrolizumab plus doxorubicin exhibited promising antitumor activity in women with advanced EC after failure to platinum therapy, even in those patients that were not MMRd.

\section{THE CLINICAL IMPACT OF MSI AND DMMR IN ADVANCED ENDOMETRIAL CANCER: THE EFFECT OF ONLINE EDUCATION ON CLINICIAN KNOWLEDGE, COMPETENCE AND CONFIDENCE}

\footnotetext{
1;2 G Fisher*, ${ }^{2} \mathrm{~A}$ Furedy, ${ }^{2} \mathrm{~J}$ Vandenbroucque, ${ }^{3} \mathrm{C}$ Marth. ${ }^{1}$ Medscape Education Global, UK;
} ${ }^{2}$ Medscape Education Global, London, UK; ${ }^{3}$ Innsbruck Medical University, Innsbruck, Austria

\subsection{6/jgc-2021-ESGO.188}

Introduction/Background* This study determined whether online continuing medical education (CME) could improve the knowledge of obstetricians/gynaecologists (obs/gyns) and oncologists (oncs) regarding the relevance of Microsatellite Instability (MSI) and deficient Mismatch Repair (dMMR) for immunotherapy paradigms in advanced endometrial cancer, and increase competence in terms of the application of appropriate diagnostic testing.

Methodology A 15-minute online video discussion between two expert faculty was launched for physicians outside the USA October 2020 with data collected to March 2021. Educational effect assessed with repeated-pairs pre-/post-activityindividual participants serving as their own control. 3 multiple-choice, knowledge questions and 1 self-efficacy, 5-point
Likert scale confidence question were analysed. McNemar's test assessed pre- to post-activity change (5\% significance level, $P$ <.05). Magnitude of change in total number of correct responses overall, and for each question, determined with Cohen's d $(<.2=$ Modest, .20-.49=Small, . $50-.79=$ Moderate, $>.80=$ Large.

Result(s)* 393 obs/gyns and 73 oncs completed pre- and postactivity questions. Positive educational effect was observed for obs/gyns (moderate effect, Cohen's $\mathrm{d}=.78, \mathrm{P}<.001$; average $\%$ of correct responses increasing from 43 to 67\%) and oncs (large effect, Cohen's $\mathrm{d}=.88, \mathrm{P}<.001$; average $\%$ of correct responses increasing from 57 to $80 \%$ ). Increases in correct responses post-activity were seen for questions on features of dMMR (\% relative improvement, obs/gyn: 37\%, oncs 25\%), appropriate diagnostic testing (obs/gyns: 17\%, oncs: 13\%), and frequency of dMMR (obs/gyns: 188\%, oncs: 133\%). The $\%$ of participants answering all questions correctly increased from 7 to $30 \%$ for obs/gyns and from 16 to $53 \%$ for oncs. Pre-activity knowledge for both specialties was low on the features and frequency of $\mathrm{dMMR}$ and despite significant improvements, there remained room for improvement postactivity. Confidence in understanding the relevance of dMMR in advanced endometrial cancer increased post-activity with $63 \%$ of obs/gyn and $48 \%$ of oncs with improved confidence. Overall, 63\% of obs/gyns and $60 \%$ of oncs improved their knowledge and competence by answering at least one more question correctly post-activity.

Conclusion* This on-demand, online video discussion resulted in a positive educational impact. However, education gaps remain evident. Online medical education is valuable in supporting the implementation of new diagnostic strategies into clinical practice as well as identifying areas of continued educational need.

\section{TUMOR CELL DISSEMINATION IS INDEPENDENT FROM ENDOMETRIAL CARCINOMA MOLECULAR SUBTYPES}

${ }^{1} \mathrm{~L}$ Volmer*, ${ }^{2} \mathrm{~S}$ Kommoss, ${ }^{1} \mathrm{C}$ Walter, ${ }^{3} \mathrm{~J}$ Mcalpine, ${ }^{4} \mathrm{~A}$ Lum, ${ }^{3} \mathrm{~A}$ Talhouk, ${ }^{1} \mathrm{~S}$ Matovina, ${ }^{1} \mathrm{~T}$ Engler, ${ }^{1} \mathrm{M}$ Grube, ${ }^{1} \mathrm{M}$ Weiss, ${ }^{5} \mathrm{~A}$ Staebler, ${ }^{1} \mathrm{~A}$ Koch, ${ }^{1} \mathrm{~A}$ Hartkopf. ${ }^{1}$ Tübingen University hospital, Department of Women's health, Tuebingen, Germany; ${ }^{2}$ Tuebingen University Hospital, Department of Women's Health, Tuebingen, Germany; ${ }^{3}$ University of British Columbia, Department of Gynecology and Obstetrics, Division of Gynecologic Oncology, Vancouver, Canada; ${ }^{4} B C$ Cancer Research Centre, Department of Molecular Oncology, Vancouver, Canada; ${ }^{5}$ Tübingen University hospital, Institute of Pathology, Tübingen, Germany

\subsection{6/ijgc-2021-ESGO.189}

Introduction/Background* Tumor cell dissemination is associated with a less favorable outcome in breast cancer patients. In contrast, only limited clinical significance was yet reported for other gynecologic malignancies. We have previously reported disseminated tumor cells (DTC) not to be associated with established risk factors, L1CAM immunoreactivity and outcome in endometrial carcinoma. It was the aim of this study to investigate potential associations of TCGA-derived molecular features such as POLE-mutation status, p53 abnormalities or MMR deficiency and the presence of DTC in the bone marrow of endometrial carcinoma patients.

Methodology Patients treated for primary endometrial carcinoma at Tuebingen University women's hospital between 2003 and 2016 with bone marrow aspirates and FFPE tumor specimens were identified. For DTC detection, cytospins were stained for pan-cytokeratin (A45-B/B3 antibody). Molecular 